\title{
HYPERSPACES HOMEOMORPHIC TO HILBERT SPACE
}

\author{
D. W. CURTIS ${ }^{1}$
}

\begin{abstract}
The hyperspace $2^{X}$ of a metric space $X$ is the space of nonempty compact subsets, topologized by the Hausdorff metric. It is shown that $2^{X}$ is homeomorphic to the separable Hilbert space $l^{2}$ if and only if $X$ is connected, locally connected, separable, topologically complete, and nowhere locally compact. The principal tool in the proof is Torunczyk's mapping characterization of $l^{2}$.
\end{abstract}

1. Hyperspace characterization theorems. For a metric space $(X, d)$, the hyperspace $2^{X}$ is the space of nonempty compact subsets of $X$, topologized by the Hausdorff metric $\tilde{d}(A, B)=\inf \{\varepsilon>0: A \subset N(B ; \varepsilon)$ and $B \subset N(A ; \varepsilon)\}$. The topology induced by $\tilde{d}$ is independent of the particular metric $d$ on $X$, since it is the same as the Vietoris finite topology on $2^{X}$. In the finite topology the basic open sets are those of the form $V\left(G_{1}, \ldots, G_{n}\right)=\left\{F \in 2^{X}: F \cap G_{i}\right.$ $\neq \varnothing$ for each $i$ and $\left.F \subset \cup_{1}^{n} G_{i}\right\}$, where $G_{1}, \ldots, G_{n}$ are open sets in $X$.

In this paper we give necessary and sufficient conditions on $X$ for the hyperspace $2^{X}$ to be homeomorphic to the separable Hilbert space $l^{2}$. This completes the following list of hyperspace characterization theorems for separable, topologically complete metric spaces.

Theorem A (Wojdyslawski [9], Tasmetov [7]). $2^{X}$ is a separable, topologically complete metric $A R$ if and only if $X$ is connected, locally connected, separable, and topologically complete.

ThEOREM B (Curtis and Schori [3], [4]). $2^{X}$ is homeomorphic to the Hilbert cube $Q=\Pi_{1}^{\infty}[-1,1]$ if and only if $X$ is nondegenerate, connected, locally connected, and compact.

TheOREM C (Curtis [2]). $2^{X}$ is homeomorphic to $Q \backslash p t \approx Q \times[0, \infty)$ if and only if $X$ is noncompact, connected, locally connected, and locally compact.

TheOREM D ([2]). $X$ admits a Peano compactification $\hat{X}$ such that the hyperspace pair $\left(2^{X}, 2^{X}\right)$ is homeomorphic to the pair $(Q, s)=\left(\Pi_{1}^{\infty}[-1,1]\right.$, $\left.\Pi_{1}^{\infty}(-1,1)\right)$ if and only if $X$ is connected, locally connected, separable, topologically complete, nowhere locally compact and admits a metric with Property S. ( $A$ metric $d$ on $X$ has Property $S$ if there exist finite covers of $X$ by connected subsets with arbitrarily small diameters.)

Received by the editors June 26, 1978 and, in revised form, August 28, 1978.

AMS (MOS) subject classifications (1970). Primary 54B20, 57A20; Secondary 54C55, 54D05.

'Supported by NSF grant MCS 76-06522. 
THEOREM E. $2^{X}$ is homeomorphic to $l^{2}$ if and only if $X$ is connected, locally connected, separable, topologically complete, and nowhere locally compact.

Since the pseudo-interior $s=\Pi_{1}^{\infty}(-1,1)$ of the Hilbert cube is homeomorphic to $l^{2}$ (Anderson [1]), Theorem D gives sufficient (but not necessary) conditions on $X$ for $2^{X}$ to be homeomorphic to $l^{2}$. The following examples illustrate the difference between (D) and (E).

EXAMPLE 1. Let $X=\cup_{1}^{n} s_{i}$, a finite union of copies of $s$, meeting at a single point. Then $X$ does admit a metric with Property $\mathrm{S}$, and the compactification $\hat{X}=\cup_{1}^{n} Q_{i}$, a union of $n$ copies of $Q$ meeting at a single point, has the property that $\left(2^{\hat{X}}, 2^{X}\right) \approx(Q, s)$. Thus in particular $2^{X} \approx s \approx l^{2}$. (This result, for $n=1$, was first proved by Kroonenberg [6.)

EXAMPLE 2. Let $X=\cup_{1}^{\infty} s_{i}$, a countably infinite union of copies of $s$ meeting at a single point $\theta$. We give $X$ the uniform topology at $\theta$ (i.e., if $f$ : $X \rightarrow s$ is a function such that the restrictions to each $s_{i}$ are homeomorphisms onto $s$, then the basic neighborhoods of $\theta$ in $X$ are of the form $f^{-1}(U)$, where $U$ is a neighborhood of $f(\theta)$ in $s$ ). This space satisfies the hypotheses of (E), but it is easy to see that there is no metric with Property S. Thus, while $X$ does have some Peano compactifications, there is no compactification $\hat{X}$ such that $\left(2^{\hat{X}}, 2^{X}\right) \approx(Q, s)$. It was shown by an ad hoc argument in [2] that $2^{X} \approx s \approx l^{2}$.

2. Torunczyk's characterization of Hilbert space. The proof of Theorem $E$ is based on the mapping characterization of $l^{2}$ given recently by Torunczyk.

DEFINITION. If o $\mathrm{l}$ is an open cover of a space $Y$, then maps $f, g: X \rightarrow Y$ are

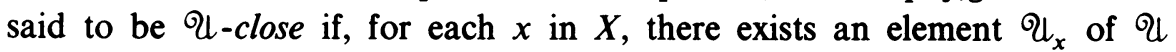
containing both $f(x)$ and $g(x)$.

Definition. A family $\left\{A_{i}\right\}$ of closed subsets in a space $Y$ is discrete if each point of $Y$ has a neighborhood meeting at most one member of $\left\{A_{i}\right\}$. (Equivalently, the family $\left\{A_{i}\right\}$ is pairwise disjoint and locally finite in $Y$.)

THEOREM ([8]). Let $Y$ be a separable, topologically complete metric AR. Then $Y$ is homeomorphic to $l^{2}$ if and only if the following condition is satisfied:

(*) For every map $f: \Sigma_{1}^{\infty} K_{i} \rightarrow Y$ of a countable disjoint sum of finite complexes, and every open cover $\mathcal{U}$ of $Y$, there exists a map $g: \Sigma_{1}^{\infty} K_{i} \rightarrow Y$ such that $f$ and $g$ are $\mathcal{Q}$-close and $\left\{g\left(K_{i}\right)\right\}$ is discrete.

3. Proof of Theorem E-Part I. Suppose $2^{X} \approx l^{2}$. Since $l^{2}$ is an AR, Theorem A implies that $X$ is connected, locally connected, separable, and topologically complete. If some point $p$ in $X$ has a compact neighborhood, then $\{p\}$ has a compact neighborhood in $2^{X}$. But this is impossible since $l^{2}$ is nowhere locally compact. Thus $X$ must also be nowhere locally compact.

Conversely, suppose $X$ satisfies the conditions of Theorem $\mathrm{E}$. Then $2^{X}$ is a separable, topologically complete metric AR, and it remains to verify the condition (*) of Torunczyk's characterization theorem. 
Let a map $f: \Sigma_{1}^{\infty} K_{i} \rightarrow 2^{X}$ and an open cover $\mathcal{U}$ of $2^{X}$ be given. For the reader's convenience, we first consider the special case in which each complex $K_{i}$ is a point and $\mathscr{U}$ is the uniform cover by $\varepsilon$-neighborhoods. (The main idea of the more general proof is particularly transparent in this case.) Thus, $f$ is simply a sequence $\left\{F_{i}\right\}$ in $2^{X}$, and we must construct a discrete sequence $\left\{G_{i}\right\}$ such that $\tilde{d}\left(F_{i}, G_{i}\right)<\varepsilon$ for each $i$.

Let $\mathcal{V}$ be a locally finite (therefore countable) open cover of $X$ with mesh $\mathcal{T}<\varepsilon$. Since $X$ is nowhere locally compact, we may choose in each element of $\mathcal{V}$ an infinite discrete sequence. The union of these sequences over all the elements of $\mathcal{V}$ is a countable discrete subset $Z$ of $X$. Each subset $G_{i}$ will be of the form $G_{i}=F_{i} \cup\left\{z_{i}\right\}$, for some $z_{i} \in Z$. The points $z_{i}$ are chosen inductively so that

(1) $d\left(z_{i}, F_{i}\right)<\varepsilon$,

(2) $z_{i} \notin \cup_{j<i} G_{j}$.

This is easily accomplished by considering any element $V_{i}$ of $\mathfrak{V}$ meeting $F_{i}$, then choosing $z_{i} \in V_{i} \cap Z \backslash \cup_{j<i} G_{j}$, which is possible since $V_{i} \cap Z$ is an infinite discrete set and $\cup_{j<i} G_{j}$ is compact. Then $\tilde{d}\left(F_{i}, G_{i}\right)=d\left(z_{i}, F_{i}\right)<\varepsilon$ for each $i$, and $G_{i} \neq G_{j}$ if $i \neq j$. If $\left\{G_{i}\right\}$ is not discrete, there exists a convergent subsequence $G_{i_{n}} \rightarrow G \in 2^{X}$. Then the sequence $\left\{z_{i_{n}}\right\}$ must cluster at some point in $G$. But $\left\{z_{i_{n}}\right\} \subset Z$ is discrete. Thus $\left\{G_{i}\right\}$ is a discrete sequence.

4. Proof of Theorem E-Part II. We now return to the general case in which each $K_{i}$ is a finite complex and $\mathcal{U}$ is an arbitrary open cover of $2^{X}$. A map $g$ : $\Sigma_{1}^{\infty} K_{i} \rightarrow 2^{X}$ must be constructed such that $f$ and $g$ are $\mathscr{U}$-close and $\left\{g\left(K_{i}\right)\right\}$ is a discrete family in $2^{X}$. This means that $g\left(K_{i}\right)$ and $g\left(K_{j}\right)$ have no common elements if $i \neq j$, and that every sequence $\left\{G_{i}\right\}$ in $2^{X}$, where each $G_{i} \in g\left(K_{i}\right)$, is discrete.

For $A \in 2^{X}$ and $\mu>0$, let $N(A ; \mu)=\{x \in X: d(x, A)<\mu\}$, and $\tilde{N}(A$; $\mu)=\left\{F \in 2^{X}: \tilde{d}(A, F)<\mu\right\}$.

Step 1. There exists a map $\mu: 2^{X} \rightarrow(0, \infty)$ such that, for each $A \in 2^{X}$, $\tilde{N}(A ; \mu(A))$ is contained in some element of $\mathcal{Q}$. (Take $\mu(A)=\frac{1}{2} \sup \{\mu>0$ : $\tilde{N}(A ; \mu) \subset U$ for some $U \in \mathcal{Q}\}$.) Thus $f, g: \sum_{1}^{\infty} K_{i} \rightarrow 2^{X}$ will be $\mathscr{U}$-close provided $\tilde{d}(f(y), g(y))<\mu f(y)$ for each $y$ in $\Sigma_{1}^{\infty} K_{i}$.

Step 2. There exists a map $\eta: 2^{X} \rightarrow(0, \infty)$ such that if $x \in N(A ; \eta(A))$, there exists an arc $J$ in $X$ connecting $x$ and $A$, with $\operatorname{diam} J<\frac{1}{2} \mu(A)$. Necessarily, $\eta(A) \leqslant \frac{1}{2} \mu(A)$. (Use the local arc-connectedness of $X$ and the compactness of elements of $2^{X}$. For $A \in 2^{X}$ define $\bar{\eta}(A)=\sup \{\eta>0$ : there exists $0<\varepsilon<\frac{1}{2} \mu(A)$ such that if $x \in N(A ; \eta)$, there exists an arc $J$ in $X$ connecting $x$ and $A$, with diam $J<\varepsilon\}$. Then $\bar{\eta}: 2^{X} \rightarrow(0, \infty)$ is lower semicontinuous, and there exists a continuous function $\eta$ such that $0<\eta(A)<$ $\bar{\eta}(A)$ for each $A \in 2^{X}$ (see [5, p. 170]).

Step 3. Subdivide each complex $K_{i}$ so that the given map $f: \Sigma_{1}^{\infty} K_{i} \rightarrow 2^{X}$ 
satisfies the following conditions for each simplex $\sigma$ :

(i) $\operatorname{diam} f(\sigma)<\frac{1}{2} \inf _{\sigma} \eta f$, where $\operatorname{diam} f(\sigma)=\sup \left\{\tilde{d}\left(f(y), f\left(y^{\prime}\right)\right): y, y^{\prime} \in \sigma\right\}$, $\boldsymbol{\sigma}\}$,

(ii) $\sup _{\sigma} \eta f<2 \inf _{\sigma} \eta f$,

(iii) $\sup _{\sigma} \mu f<\frac{3}{2} \inf _{\sigma} \mu f$.

Step 4. For each $n$, let $\mathcal{V}_{n}$ be a locally finite (therefore countable) open cover of $X$ with mesh $\mathfrak{V}_{n}<1 / n$. In each element of $\mathfrak{V}_{n}$ choose an infinite discrete subset of $X$. Then the union of such subsets over all the elements of $\mathcal{V}_{n}$ is an infinite discrete subset $Z(n)$ of $X$.

Step 5. We define $g$ first at each vertex of the complex $K_{1}$. For a vertex $v$ let $n_{v}$ be the smallest integer such that $1 / n_{v}<\frac{1}{4} \eta f(v)$, and choose a point $z_{v}$ in $Z\left(n_{v}\right)$ such that $d\left(z_{v}, f(v)\right)=\min \left\{d\left(z_{v}, x\right): x \in f(v)\right\}<1 / n_{v}$. Set $g(v)=f(v)$ $\cup\left\{z_{v}\right\}$.

Step 6. We now extend $g$ over the 1-skeleton $K_{1}^{(1)}$ of $K_{1}$. Let $\tau$ be an edge of $K_{1}$, with endpoints $v$ and $w$ and midpoint $\tau^{*}$. Since

$$
\begin{aligned}
d\left(z_{v}, f\left(\tau^{*}\right)\right) & \leqslant d\left(z_{v}, f(v)\right)+\tilde{d}\left(f(v), f\left(\tau^{*}\right)\right) \leqslant \frac{1}{4} \eta f(v)+\frac{1}{2} \inf _{\tau} \eta f \\
& \leqslant \frac{1}{2} \inf _{\tau} \eta f+\frac{1}{2} \inf _{\tau} \eta f \leqslant \eta f\left(\tau^{*}\right),
\end{aligned}
$$

there exists an $\operatorname{arc} J_{v}$ (possibly degenerate) in $X$ connecting $z_{v}$ and $f\left(\tau^{*}\right)$, with $\operatorname{diam} J_{v}<\frac{1}{2} \mu f\left(\tau^{*}\right)$. Likewise, there exists an arc $J_{w}$ connecting $z_{w}$ and $f\left(\tau^{*}\right)$, with $\operatorname{diam} J_{w}<\frac{1}{2} \mu f\left(\tau^{*}\right)$. Define $g\left(\tau^{*}\right)=f\left(\tau^{*}\right) \cup J_{v} \cup J_{w^{*}}$ Then $\tilde{d}\left(f\left(\tau^{*}\right)\right.$, $\left.g\left(\tau^{*}\right)\right)<\frac{1}{2} \mu f\left(\tau^{*}\right)$.

Let $h: I \rightarrow 2^{X}$ be a path from $f\left(\tau^{*}\right)$ to $g\left(\tau^{*}\right)$ such that $h(0)=f\left(\tau^{*}\right)$, $h(1)=g\left(\tau^{*}\right)$, and $f\left(\tau^{*}\right) \subset h(t) \subset g\left(\tau^{*}\right)$ for each $t$. Define $g$ on the segment $\left[v \tau^{*}\right]$ (and similarly on the segment $\left[w \tau^{*}\right]$ ) as follows:

$$
g\left((1-t) v+t \tau^{*}\right)= \begin{cases}f\left((1-2 t) v+2 t \tau^{*}\right) \cup\left\{z_{v}\right\} & \text { if } 0 \leqslant t \leqslant 1 / 2, \\ h(2 t-1) \cup\left\{z_{v}\right\} & \text { if } 1 / 2 \leqslant t \leqslant 1 .\end{cases}
$$

Thus $g$ is defined over each edge $\tau=\left[v \tau^{*}\right] \cup\left[w \tau^{*}\right]$. We claim that for each $p \in \tau, \tilde{d}\left(f\left(\tau^{*}\right), g(p)\right) \leqslant \frac{1}{2} \mu f\left(\tau^{*}\right)$. With $p=(1-t) v+t \tau^{*}$, suppose first that $0<t<1 / 2$. Then

$$
\tilde{d}\left(f\left(\tau^{*}\right), f\left((1-2 t) v+2 t \tau^{*}\right)\right) \leqslant \operatorname{diam} f(\tau)<\frac{1}{2} \inf _{\tau} \eta f<\frac{1}{2} \eta f\left(\tau^{*}\right),
$$

and as shown above, $d\left(z_{v}, f\left(\tau^{*}\right)\right) \leqslant \eta f\left(\tau^{*}\right)$. It follows that $\tilde{d}\left(d\left(\tau^{*}\right), f((1-2 t) v\right.$ $\left.\left.+2 t \tau^{*}\right) \cup\left\{z_{v}\right\}\right) \leqslant \eta f\left(\tau^{*}\right) \leqslant \frac{1}{2} \mu f\left(\tau^{*}\right)$. Now suppose $\frac{1}{2} \leqslant t<1$. Then $f\left(\tau^{*}\right) \subset$ $g(p) \subset g\left(\tau^{*}\right)$, hence $\tilde{d}\left(f\left(\tau^{*}\right), g(p)\right)<\tilde{d}\left(f\left(\tau^{*}\right), g\left(\tau^{*}\right)\right)<\frac{1}{2} \mu f\left(\tau^{*}\right)$.

Step 7. Extend $g$ over all of $K_{1}$ as follows. Let $\mathcal{C}_{1}$ denote the hyperspace of subcontinua of the 1-skeleton $K_{1}^{(1)}$. There exists a map $r: K_{1} \rightarrow \mathcal{C}_{1}$ such that $r(p)=\{p\}$ for each $p$ in $K_{1}^{(1)}$, and such that if $\sigma$ is the carrier of the point $y$, then $r(y) \subset \sigma^{(1)}$. (First define $r$ over the 2-skeleton $K_{1}^{(2)}$ as follows. Consider each 2 -simplex $\sigma$ as the cone over its boundary $\sigma^{(1)}$, with cone point $c$. For each point $y=t c+(1-t) p$ in $\sigma$, where $p \in \sigma^{(1)}$, take $r(y)$ to be a subcon- 
tinuum of $\sigma^{(1)}$ centered at $p$ and with length proportional to $t$. If $t=1$, then $r(y)=r(c)=\sigma^{(1)}$. Similarly, $r$ is extended inductively over the higherdimensional skeleta.) Now define $g(y)=\bigcup\{g(p): p \in r(y)\}$.

We claim that $\tilde{d}(f(y), g(y)) \leqslant \mu f(y)$ for each $y$ in $K_{1}$. Let $\sigma$ be the carrier of $y$, and set $F_{y}=\bigcup\left\{f\left(\tau^{*}\right): \tau\right.$ is an edge of $\sigma$ meeting $\left.r(y)\right\}$. Then $\tilde{d}(f(y)$, $g(y)) \leqslant \tilde{d}\left(f(y), F_{y}\right)+\tilde{d}\left(F_{y}, g(y)\right) \leqslant \frac{1}{2} \inf _{\sigma} \eta f+\frac{1}{2} \sup _{o} \mu f \leqslant \frac{1}{4} \inf _{o} \mu f$ $+\frac{3}{4} \inf _{\sigma} \mu f \leqslant \mu f^{\prime}(y)$.

Thus $g: K_{1} \rightarrow 2^{X}$ is $\mathcal{U}$-close to the restriction $f: K_{1} \rightarrow 2^{X}$, and for each $y$ in $K_{1}$ with carrier $\sigma, g(y)$ contains the point $z_{v}$ in $Z\left(n_{v}\right)$ for at least one vertex $v$ of $\sigma$. Replacing $g(y)$ by $f(y) \cup g(y)$, we may assume also that $f(y) \subset g(y)$ for each $y$.

Step 8. The map $g$ is defined similarly over each complex $K_{i}$, with the stipulation that the points $z_{v}$ are chosen from $Z\left(n_{v}\right) \backslash \cup\left\{g(y): y \in K_{1}\right.$ $\left.\cup \cdots \cup K_{i-1}\right\}$. This insures that the images $g\left(K_{1}\right), g\left(K_{2}\right), \ldots$ in $2^{X}$ are disjoint.

We claim that $\left\{g\left(K_{i}\right)\right\}$ is a discrete family in $2^{X}$. Suppose not. Then there exists a convergent sequence $g\left(y_{n_{i}}\right) \rightarrow G \in 2^{X}$, where $y_{n_{i}} \in K_{n_{i}}$ for each $i$. For convenience assume $n_{i}=i$; thus $g\left(y_{i}\right) \rightarrow G$. Each $g\left(y_{i}\right)$ contains a point $z_{i}$ of $Z\left(n_{v_{i}}\right)$, for some vertex $v_{i}$ of the carrier $\sigma_{i}$ of $y_{i}$. Since every finite union $\cup_{n=1}^{k} Z(n)$ is a discrete subset of $X$, and since every subsequence of $\left\{z_{i}\right\}$ must cluster at some point of $G$, we must have $n_{v_{i}} \rightarrow \infty$. Since $n_{v_{i}}$ was specified to be the smallest integer for which $1 / n_{v_{i}}<\frac{1}{4} \eta f\left(v_{i}\right)$, it follows that $\eta f\left(v_{i}\right) \rightarrow 0$. Since $f\left(y_{i}\right) \subset g\left(y_{i}\right)$ for each $i$, some subsequence $\left\{f\left(y_{i_{j}}\right)\right\}$ must converge to a subset $F$ of $G$. Thus $\eta f\left(y_{i}\right) \rightarrow \eta(F)>0$. But then $\eta f\left(v_{i_{j}}\right) \geqslant \inf _{\sigma_{i, j}} \eta f>\frac{1}{2} \sup _{\sigma_{i j}} \eta f \geqslant \frac{1}{2} \eta f\left(y_{i_{j}}\right) \rightarrow \frac{1}{2} \eta(F)>0$, hence $\eta f\left(v_{i}\right) \nrightarrow 0$, a contradiction.

\section{REFERENCES}

1. R. D. Anderson, Hilbert space is homeomorphic to the countable infinite product of lines, Bull. Amer. Math. Soc. 72 (1966), 515-519.

2. D. W. Curtis, Hyperspaces of noncompact metric spaces, Compositio Math. (to appear).

3. D. W. Curtis and R. M. Schori, $2^{X}$ and $C(X)$ are homeomorphic to the Hilbert cube, Bull. Amer. Math. Soc. 80 (1974), 927-931.

4. Hyperspaces of Peano continua are Hilbert cubes, Fund. Math. 101 (1978), 19-38.

5. J. Dugundji, Topology, Allyn and Bacon, Boston, Mass., 1966.

6. N. Kroonenberg, Pseudo-interiors of hyperspaces, Compositio Math. 32 (1976), 113-131.

7. U. Tasmetov, On the connectedness of hyperspaces, Soviet Math. Dokl. 15 (1974), 502-504.

8. H. Torunczyk, Characterizing Hilbert space topology (preprint).

9. M. Wojdyslawski, Retractes absolus et hyperespaces des continus, Fund. Math. 32 (1939), $184-192$.

Department of Mathematics, Louisiana State University, Baton Rouge, Louisiana 70803 